\title{
Research Article Asymptotic Stability of Semi-Markov Modulated Jump Diffusions
}

\author{
Amogh Deshpande \\ Department of Applied Finance and Actuarial Studies, Faculty of Business and Economics, \\ Macquarie University, Sydney, NSW 2109, Australia \\ Correspondence should be addressed to Amogh Deshpande, addeshpa@gmail.com
}

Received 28 February 2012; Revised 6 July 2012; Accepted 12 July 2012

Academic Editor: Lukasz Stettner

Copyright (C 2012 Amogh Deshpande. This is an open access article distributed under the Creative Commons Attribution License, which permits unrestricted use, distribution, and reproduction in any medium, provided the original work is properly cited.

\begin{abstract}
We consider the class of semi-Markov modulated jump diffusions (sMMJDs) whose operator turns out to be an integro-partial differential operator. We find conditions under which the solutions of this class of switching jump-diffusion processes are almost surely exponentially stable and moment exponentially stable. We also provide conditions that imply almost sure convergence of the trivial solution when the moment exponential stability of the trivial solution is guaranteed. We further investigate and determine the conditions under which the trivial solution of the sMMJDperturbed nonlinear system of differential equations $d X_{t} / d t=f\left(X_{t}\right)$ is almost surely exponentially stable. It is observed that for a one-dimensional state space, a linear unstable system of differential equations when stabilized just by the addition of the jump part of an sMMJD process does not get destabilized by any addition of a Brownian motion. However, in a state space of dimension at least two, we show that a corresponding nonlinear system of differential equations stabilized by jumps gets destabilized by addition of Brownian motion.
\end{abstract}

\section{Introduction}

The stability of stochastic differential equations (SDEs) has a long history with some key works being those of Arnold [1], Khasminskii [2], and Ladde and Lakshmikantham [3]. SDEs with switching have been applied in diverse areas such as finance (Deshpande and Ghosh [4]) and biology (Hanson [5]). On the same note, the stability of these processes has been much studied, in particular by Ji and Chizeck [6] and Mariton [7], who both studied the stability of a jump-linear system of the form $\dot{x}_{t}=A\left(r_{t}\right) x_{t}$, where $r_{t}$ is a Markov chain. Basak et al. [8] discussed the stability of a semilinear SDE with Markovian-regime switching of the form $\dot{x}_{t}=A\left(r_{t}\right) x_{t} d t+\sigma\left(r_{t}, x_{t}\right) d W_{t}$. Mao [9] studied the exponential stability of a general nonlinear diffusion with Markovian switching of the form $d x_{t}=f\left(x_{t}, t, r_{t}\right) d t+$ $g\left(x_{t}, t, r_{t}\right) d W_{t}$. Yin and $\mathrm{Xi}$ [10] studied the stability of Markov-modulated jump-diffusion processes (MMJDs). 
Consider the following jump-diffusion equation in which the coefficients are modulated by an underlying semi-Markov process:

$$
\begin{gathered}
d X_{t}=b\left(X_{t}, \theta_{t}\right) d t+\sigma\left(X_{t}, \theta_{t}\right) d W_{t}+d J_{t}, \\
d J_{t}=\int_{\Gamma} g\left(X_{t}, \theta_{t}, \gamma\right) N(d t, d \gamma), \\
X_{0}=x, \quad \theta_{0}=i,
\end{gathered}
$$

where $X(\cdot)$ takes values in $\mathbb{R}^{r}$ and $\theta_{t}$ is a finite-state semi-Markov process taking values in $\mathcal{X}=\{1, \ldots, M\}$. Let $\Gamma$ be a subset of $\mathbb{R}^{r}-0$; it is the range space of impulsive jumps. For any set $B$ in $\Gamma, N(t, B)$ counts the number of jumps on $[0, t]$ with values in $B$ and is independent of the Brownian motion $W_{t}, b(\cdot, \cdot): \mathbb{R}^{r} \times \mathcal{X} \rightarrow \mathbb{R}^{r}, \sigma(\cdot, \cdot): \mathbb{R}^{r} \times \mathcal{X} \rightarrow \mathbb{R}^{r} \times \mathbb{R}^{d}, g(\cdot, \cdot, \cdot)$ : $\mathbb{R}^{r} \times \mathcal{X} \times \Gamma \rightarrow \mathbb{R}^{r}$. For future use we define the compensated Poisson measure $\widetilde{N}(d t, d \gamma)=$ $N(d t, d \gamma)-\lambda \pi(d \gamma) d t$, where $\pi(\cdot)$ is the jump distribution and $0<\lambda<\infty$ is the jump rate. Equation (1.1) can be regarded as the result of the following $M$ equations:

$$
\begin{gathered}
d X_{t}=b\left(X_{t}, i\right) d t+\sigma\left(X_{t}, i\right) d W_{t}+\int_{\Gamma} g\left(X_{t}, i, \gamma\right) N(d t, d \gamma) \\
X_{0}=x, \quad \theta_{0}=\theta
\end{gathered}
$$

which switch from one state to another according to the underlying movement of the semiMarkov process.

Unlike the special Markov-modulated case in which the $x$-dependent diffusion is a partial differential operator, the semi-Markov case is characterized by an integro-partial differential operator. In this paper we study the asymptotic stability of sMMJDs. We also investigate the perturbation of the nonlinear differential equation $d X_{t} / d t=f\left(X_{t}\right)$ by an sMMJD. We determine the conditions under which the perturbed system is almost surely exponentially stable. We show that for a one-dimensional state space, the deterministic linear unstable system of differential equations that can be stabilized by the addition of a jump component of the process $X_{t}$, surprisingly can never be destabilized by an addition of a Brownian motion. An interesting question we may ask here is, can the similar inference hold true for $X_{t}$ in higher dimension? The answer is surprisingly no. We show that for a state space with dimension greater than or equal to 2 , a corresponding nonlinear system that is stabilized by the jump component of the process $X_{t}$ can in fact be destabilized by addition of the Brownian motion part. We organize the paper as follows.

In Section 2, we briefly establish a representation of a class of semi-Markov processes as a stochastic integral with respect to a Poisson random measure. We define the concepts of almost sure exponential stability and moment exponential stability. In Section 3, we present conditions that guarantee almost sure exponential stability and moment exponential stability of the trivial solution of (1.1). In general, there is no connection between these two stability criteria. However, under additional conditions one can say when does the moment exponential stability guarantees or implies almost sure exponential stability. We elaborate on this aspect while concluding this section. In Section 4, we provide some examples to illustrate these two stability criterion in our context. In Section 5, we investigate the conditions for which a nonlinear system of differential equation of the type $d X_{t} / d t=f\left(X_{t}\right)$ is almost surely 
exponentially stable. We then investigate its behavior in higher-dimensional state space, as mentioned earlier. The paper ends with concluding remarks.

\section{Preliminaries}

We assume that the probability space $\left(\Omega, \mathcal{F},\left\{\mathcal{F}_{t}\right\}, \mathbb{P}\right)$ is complete with filtration $\left\{\mathcal{F}_{t}\right\}_{t \geq 0}$ and is right-continuous and $\mathcal{F}_{0}$ contains all $\mathbb{P}$ null sets. If $v$ is some vector, then $|v|$ is its Euclidean norm and $v^{\prime}$ is its transpose, while if $A$ is a matrix then its trace norm is denoted as $\|A\|=\sqrt{\operatorname{tr}\left(A^{\prime} A\right)} \cdot \mathbb{R}_{+}$stands for positive part of the real line while $r$ is a positive integer. Let $\mathcal{C}^{2,1}\left(\mathbb{R}^{r} \times \mathcal{X} \times \mathbb{R}_{+}\right)$denote the family of all functions on $\mathbb{R}^{r} \times \mathcal{X} \times \mathbb{R}_{+}$, which are twice continuously differentiable in $x$ and continuously differentiable in $y$. Consider $\left\{\theta_{t}\right\}_{t \geq 0}$ as a semi-Markov process taking values in $\chi \mathcal{L}$ with transition probability $p_{i, j}$ and conditional holding time distribution $F(t \mid i)$. Thus if $0 \leq t_{0} \leq t_{1} \leq \cdots$ are times when jumps occur, then

$$
P\left(\theta_{t_{n+1}}=j, t_{n+1}-t_{n} \leq t \mid \theta_{t_{n}}=i\right)=p_{i j} F(t \mid i) .
$$

Matrix $\left[p_{i j}\right]_{\{i, j=1, \ldots, M\}}$ is irreducible and for each $i, F(\cdot \mid i)$ has continuously differentiable and bounded density $f(\cdot \mid i)$. Embed $\mathcal{X}$ in $\mathbb{R}^{r}$ by identifying $i$ with $e_{i} \in \mathbb{R}^{r}$. For $y \in[0, \infty) i, j \in \mathcal{X}$, let

$$
\lambda_{i j}(y)=p_{i j} \frac{f(y / i)}{1-F(y / i)} \geq 0, \quad \forall i \neq j, \quad \lambda_{i i}(y)=-\sum_{j \in \mathcal{X}, j \neq i} \lambda_{i j}(y) \quad \forall i \in \mathcal{X} .
$$

Let the stationary distribution of the semi-Markov process be defined as $v_{i} \triangleq(1 / t) \int_{0}^{t} \mathbb{I}_{\theta_{s}=i} d s$ where $\mathbb{I}$. takes value 1 if $\theta_{s}=i$ and 0 otherwise for any $i \in \mathcal{X}$.

For $i \neq j \in \mathcal{X}, y \in \mathbb{R}_{+}$, let $\Lambda_{i j}(y)$ be consecutive (with respect to lexicographic ordering on $\mathcal{X} \times \mathcal{X}$ ) left-closed, right-open intervals of the real line, each having length $\lambda_{i j}(y)$. Define the functions $\bar{h}: \mathcal{X} \times \mathbb{R}_{+} \times \mathbb{R} \rightarrow \mathbb{R}$ and $\bar{g}: \mathcal{X} \times \mathbb{R}_{+} \times \mathbb{R} \rightarrow \mathbb{R}_{+}$by

$$
\begin{gathered}
\bar{h}(i, y, z)= \begin{cases}j-i & \text { if } z \in \Lambda_{i j}(y), \\
0 & \text { otherwise, }\end{cases} \\
\bar{g}(i, y, z)= \begin{cases}y & \text { if } z \in \Lambda_{i j}(y), j \neq i, \\
0 & \text { otherwise. }\end{cases}
\end{gathered}
$$

Let $\mathcal{M}\left(\mathbb{R}_{+} \times \mathbb{R}\right)$ be the set of all nonnegative integer-valued $\sigma$-finite measures on a Borel $\sigma$-field of $\left(\mathbb{R}_{+} \times \mathbb{R}\right)$. Define the process $\left\{\theta_{t}^{\prime}, Y_{t}\right\}$ described by the following stochastic integral equations:

$$
\begin{aligned}
& \theta_{t}^{\prime}=\theta_{0}^{\prime}+\int_{0}^{t} \int_{\mathbb{R}} \bar{h}\left(\theta_{u^{-}}, Y_{u^{-}}, z\right) N_{1}(d u, d z) \\
& Y_{t}=t-\int_{0}^{t} \int_{\mathbb{R}} \bar{g}\left(\theta_{u^{-}}, Y_{u^{-}}, z\right) N_{1}(d u, d z)
\end{aligned}
$$


where $N_{1}(d t, d z)$ is an $\mathcal{M}\left(\mathbb{R}_{+} \times \mathbb{R}\right)$-valued Poisson random measure with intensity $d t m(d z)$ independent of the $\mathcal{X}$-valued random variable $\theta_{0}^{\prime}$, where $m(\cdot)$ is a Lebesgue measure on $\mathbb{R}$. We define the corresponding compensated or centred Poisson measure as $\widetilde{N}_{1}(d s, d z)=$ $N_{1}(d s, d z)-d s m(d z)$. It was shown in Theorem 2.1 of Ghosh and Goswami [11] that $\theta_{t}^{\prime}$ is a semi-Markov process with transition probability matrix $\left[p_{i j}\right]_{\{i, j=1, \ldots, M\}}$ with conditional holding time distributions $F(y \mid i)$. Therefore, one can write $\theta_{t}^{\prime}=\theta_{t}$. We assume that $N(\cdot, \cdot), N_{1}(\cdot, \cdot)$, and $\theta_{0}, W_{t}, S_{0}$ defined on $(\Omega, \mp, \mathbb{P})$ are independent.

To ensure that zero is the only equilibrium point of (1.1), we need the following assumption.

Assumption 2.1. Assume $g(x, i, \gamma)$ is $\mathbb{B}\left(\mathbb{R}^{r} \times \mathcal{X} \times(\mathbb{R}-\{0\})\right)$-measurable and that constants $C>0$ exist such that for each $i \in \mathcal{X}, x_{1}, x_{2}$ being $\mathbb{R}^{r}$-valued and for each $\gamma \in \Gamma$ we have

$$
\begin{gathered}
\left|b\left(x_{1}, i\right)-b\left(x_{2}, i\right)\right|+\left|\sigma\left(x_{1}, i\right)-\sigma\left(x_{2}, i\right)\right| \leq C\left|x_{1}-x_{2}\right| \\
\left|g\left(x_{1}, i, \gamma\right)-g\left(x_{2}, i, \gamma\right)\right| \leq C\left|x_{1}-x_{2}\right| .
\end{gathered}
$$

We also need the condition that the generator matrix $Q(\cdot)$ is bounded and continuous. $b(0, i)=$ $0, \sigma(0, i)=0$ and $g(x, i, 0)=0$ and $g(0, i, \gamma)=0$ for each $x \in \mathbb{R}^{r}, i \in \mathcal{X}$ and each $\gamma \in \Gamma$.

The process $\left(X_{t}, \theta_{t}, Y_{t}\right)$ defined on $(\Omega, \mathcal{F}, \mathbb{P})$ in $(1.1)$ and (2.4) is jointly Markov and has a generator $G$ given as follows. For $f \in \mathcal{C}^{2,1}\left(\mathbb{R}^{r}, \mathcal{X}, \mathbb{R}_{+}\right)$, we have

$$
\begin{aligned}
G f(x, i, y)= & \frac{1}{2} \sum_{k, l=1}^{r} a_{k l}(x, i) \frac{\partial f(x, i, y)}{\partial x_{k} \partial x_{l}}+\sum_{k=1}^{r} b_{k}(x, i) \frac{\partial f(x, i, y)}{\partial x_{k}} \\
& +\frac{\partial f(x, i, y)}{\partial y}+\frac{f(y \mid i)}{1-F(y \mid i)} \sum_{j \neq i, j \in x} p_{i j}[f(x, j, 0)-f(x, i, y)] \\
& +\lambda \int_{\Gamma}(f(x+g(x, i, \gamma), i, y)-f(x, i, y)) \pi(d \gamma)
\end{aligned}
$$

where $x \in \mathbb{R}^{r}, a(x, i)=\sigma(x, i) \sigma^{\prime}(x, i)$ is an $\mathbb{R}^{r \times r}$ matrix and $a_{k l}(x, i)$ is the $(k, l)$ th element of the matrix $a$ while $b_{k}(x, i)$ is the $k$ th element of the vector $b(x, i)$.

We define the jump times, that is, time epochs when jumps occur by $\left\{\tau_{n}^{N}\right\}$, where $\tau_{1}^{N}<\tau_{2}^{N}<\cdots<\tau_{n}^{N}<\cdots$, to be the enumeration of all elements in the domain $D_{p}$ of the point process $p(t)$ corresponding to the stationary $\mathcal{F}_{t}$-Poisson point process $N(d t, d \gamma)$. It is easy to see that $\left\{\tau_{n}^{N}\right\}$ is an $\mathscr{F}_{t}$-stopping time for each $n$. Moreover, we have $\lim _{n \rightarrow \infty} \tau_{n}^{N}=+\infty$ since the characteristic measure $m(\cdot)$ is finite. Next, let us denote the successive switching instants of the second component, which is the semi-Markov process $\theta_{t}$ that switches from one point on the space $\mathcal{X}$ to another and is denoted by $\tau_{0}^{\theta}=0, \tau_{n}^{\theta}=\inf \left\{t: t>\tau_{n-1}^{\theta}, X_{t} \neq X_{\tau_{n-1}^{\theta}}\right\}, n \geq 1$. Since the Poisson random measure $N(\cdot, \cdot)$ is independent of $N_{1}(\cdot, \cdot)$, one could adapt the proof of $\mathrm{Xi}$ ([12]) to show that with probability $1,\left\{\tau_{n}^{N}: n \geq 1\right\}$ and $\left\{\tau_{n}^{\theta}: n \geq 1\right\}$ are mutually disjoint. Hence between two chain-switching epochs, the process $X_{t}$ behaves like an ordinary jumpdiffusion process without switching, a fact that we will use below to show the existence and uniqueness of the sMMJD process $X_{t}$. Accordingly, we describe next the existence-uniqueness theorem for (1.1). 
Theorem 2.2. Assume that Assumption 2.1 holds. Then there exists a unique solution $\left(X_{t}, t \geq 0\right)$ with initial data $\left(X_{0}, \theta_{0}, Y_{0}\right)$ to (1.1).

Proof. We only provide a sketch of the proof here. Consider $[s, t], \tau_{1}^{\theta}, \ldots, \tau_{N}^{\theta} \leq t$. Then as described above, on each of the intervals between the chain switching times, that is, $\left[s, \tau_{1}^{\theta}\right)$, $\ldots,\left(\tau_{N}^{\theta}, t\right]$, the sMMJD process $X_{t}$ behaves like a jump-diffusion process. We can then use the standard Picard iteration argument in Applebaum [13] to show the existence-uniqueness of solution $X_{t}$.

Before we proceed with our main analysis concerning these two stability issues we introduce a key Lemma.

Lemma 2.3. $\left\{P\left(X_{t} \neq 0, t \neq 0\right)\right\}=1$ for any $X_{0}=x \neq 0$, and $\theta_{0}=\theta \in \chi$. Thus almost all sample paths of any solutions of (1.1) starting from a nonzero state will never reach the origin.

Proof. We show this in a simple way. From the condition on the coefficients, $b(0, i)=0$, $\sigma(0, i)=0$, and $g(0, i, 0)=0$. So $(1.1)$ admits a trivial solution $X_{t}=0$. From Theorem 2.2 above, due to the uniqueness of the solution of (1.1) the conclusion now follows.

We next have the following generalized Ito's formula.

Lemma 2.4. Utilizing the operator $G$ in (2.1), the generalized Ito's formula is given by

$$
\begin{aligned}
& f\left(X_{t}, \theta_{t}, Y_{t}\right)-f(x, \theta, y)= \int_{0}^{t} G f\left(X_{s}, \theta_{s}, Y_{s}\right) d s+\int_{0}^{t}\left(\nabla f\left(X_{s}, \theta_{s}, Y_{s}\right)\right)^{\prime} \sigma\left(X_{s}, \theta_{s}\right) d W_{s} \\
&+\int_{0}^{t} \int_{\Gamma}\left[f\left(X_{s-}+g\left(X_{s^{-}}, \theta_{s^{-}}, \gamma\right), \theta_{s}, Y_{s-}\right)-f\left(X_{s^{-}}, \theta_{s^{-}}, Y_{s-}\right)\right] \widetilde{N}(d s, d \gamma) \\
&+\int_{0}^{t} \int_{\mathbb{R}}\left[f \left(X_{s^{-}}, \theta_{s^{-}}+\bar{h}\left(\theta_{s^{-}}, Y_{s^{-}}, z\right),\right.\right. \\
&\left.\left.Y_{s^{-}}-\bar{g}\left(\theta_{s^{-}}, Y_{s^{-}}, z\right)\right)-f\left(X_{s^{-}}, \theta_{s^{-}}, Y_{s^{-}}\right)\right] \widetilde{N}_{1}(d s, d z),
\end{aligned}
$$

where the local martingale terms are explicitly defined as

$$
\begin{gathered}
d M_{1}(t):=\left(\nabla f\left(X_{t}, \theta_{t}, Y_{t}\right)\right)^{\prime} \sigma\left(X_{t}, \theta_{t}\right) d W_{t} \\
d M_{2}(t):=\int_{\Gamma}\left[f\left(X_{s-}+g\left(X_{s^{-}}, \theta_{s-}, \gamma\right), \theta_{s-}, Y_{s-}\right)-f\left(X_{s^{-}}, \theta_{s-}, Y_{s-}\right)\right] \widetilde{N}(d s, d \gamma), \\
d M_{3}(t):=\int_{\mathbb{R}}\left[f\left(X_{s^{-}}, \theta_{s-}+\bar{h}\left(\theta_{s^{-}}, Y_{s^{-}}, z\right), Y_{s^{-}}-\bar{g}\left(\theta_{s^{-}}, Y_{s^{-}}, z\right)\right)-f\left(X_{s^{-}}, \theta_{s^{-}}, Y_{s-}\right)\right] \widetilde{N}_{1}(d s, d z) .
\end{gathered}
$$

Proof. For details refer to Ikeda and Watanabe [14].

We now discuss the two criteria for stochastic stability that we intend to consider. 
Definition 2.5 (Almost sure exponential stability). The trivial solution of (1.1) is almost surely exponentially stable if

$$
\limsup _{t \rightarrow \infty} \frac{1}{t} \log \left|X_{t}\right|<0 \quad \text { a.s. } \forall X_{0} \in \mathbb{R}^{r} \text { a.s. }
$$

The quantity on the left-hand side of the above equation is termed as the sample Lyapunov exponent.

Definition 2.6 (Moment exponential stability). Let $p>0$. The trivial solution of (1.1) is said to be $p$ th moment exponentially stable if there exists a pair of constants $\lambda>0$ and $C>0$, such that for any $X_{0} \in \mathbb{R}^{r}$

$$
E\left[\left|X_{t}\right|^{p}\right] \leq C\left|X_{0}\right|^{p} \exp (-\lambda t) \quad \forall t \leq 0
$$

In the next section, we detail the proofs for obtaining the conditions under which the trivial solution of (1.1) is almost surely exponentially stable and moment exponentially stable.

\section{Almost Sure Stability and Moment Exponential Stability}

In the sequel we will always, as standing hypotheses, assume that Assumption 2.1 holds. From Theorem 2.2 we deduce that there exists a unique solution to (1.1). By Lemma 2.3, we know that $X_{t}$ will never reach zero whenever $X_{0} \neq 0$. So in what follows we will only need a function $V(x, i, y) \in \mathcal{C}^{2,1}\left(\mathbb{R}^{r} \times \mathcal{X} \times \mathbb{R}_{+}\right)$defined on the domain of the deleted neighborhood of zero. Our first main result provides conditions under which the trivial solution to (1.1) is almost surely exponentially stable.

Theorem 3.1. Assume that there exist a function $V \in \mathcal{C}^{2,1}\left(\mathbb{R}^{r} \times \mathcal{X} \times \mathbb{R}_{+}\right)$in any deleted neighborhood of zero. Moreover, assume that there exist positive constants $\alpha, \beta, \rho_{1}, \rho_{2}, \bar{\rho}_{1}$, and $\bar{\rho}_{2}$ for each $x \in$ $\mathbb{R}^{r}, i \in \mathcal{X}$ and for each $\gamma \in \Gamma$ such that

$$
\begin{gathered}
G \log V(x, i, y) \leq-\alpha, \\
\left|\left(\nabla_{x} V(x, i, y)\right)^{\prime} \sigma(x, i)\right| \leq \beta V(x, i, y), \\
\rho_{1} \leq\left(\frac{V(x+g(x, i, \gamma), i, y)}{V(x, i, y)}\right) \leq \rho_{2}, \\
\bar{\rho}_{1} \leq\left(\frac{V(x, i+\bar{h}(i, y, z), y-\bar{g}(i, y, z))}{V(x, i, y)}\right) \leq \bar{\rho}_{2},
\end{gathered}
$$

then the solution to (1.1) is almost surely exponentially stable. 
Proof. Note that

$$
\begin{aligned}
\log V\left(X_{t}, \theta_{t}, Y_{t}\right)= & \log V\left(X_{0}, \theta_{0}, Y_{0}\right)+\int_{0}^{t} G \log V\left(X_{s}, \theta_{s}, Y_{s}\right) d s \\
& +M_{1}(t)+M_{2}(t)+M_{3}(t) .
\end{aligned}
$$

Here the local martingale terms $M_{1}(t), M_{2}(t)$, and $M_{3}(t)$ are, respectively,

$$
\begin{gathered}
M_{1}(t)=\int_{0}^{t} \frac{\left(\nabla_{x} V\left(X_{s}, \theta_{s}, Y_{s}\right)\right)^{\prime} \sigma\left(X_{s}, \theta_{s}\right)}{V\left(X_{s}, \theta_{s}, Y_{s}\right)} d W_{s} \\
M_{2}(t)=\int_{0}^{t} \int_{\Gamma} \log \left(\frac{V\left(X_{s-}+g\left(X_{s-}, \theta_{s^{-}}, \gamma\right), \theta_{s-}, Y_{s-}\right)}{V\left(X_{s^{-}}, \theta_{s^{-}}, Y_{s-}\right)}\right) \widetilde{N}(d s, d \gamma) \\
M_{3}(t)=\int_{0}^{t} \int_{\mathbb{R}}\left[\log V\left(X_{s-}, \theta_{s^{-}}+\bar{h}\left(\theta_{s^{-}}, Y_{s-}, z\right), Y_{s^{-}}-\bar{g}\left(\theta_{s^{-}}, Y_{s^{-}}, z\right)\right)\right. \\
\left.-\log V\left(X_{s^{-}}, \theta_{s^{-}}, Y_{s^{-}}\right)\right] \widetilde{N}_{1}(d s, d z) .
\end{gathered}
$$

We deal with (3.2) term by term to derive an upper bound on $\lim \sup _{t \rightarrow \infty} \log V\left(X_{t}, i, Y_{t}\right) / t$. Consider first the drift term of (3.2). It is easy to see from the assumptions made that $\int_{0}^{t} G \log V\left(X_{s}, \theta_{s}, Y_{s}\right) d s$ will be bounded above by $-\alpha t$. Secondly, we now concentrate on the local martingale terms of (3.2). First consider the quadratic variation of the $M_{1}(t)$ term. By Ito's isometry, we have

$$
\begin{aligned}
\left\langle M_{1}(t), M_{1}(t)\right\rangle & =\int_{0}^{t}\left|\frac{\left(\nabla_{x} V\left(X_{s}, \theta_{s}, Y_{s}\right)\right)^{\prime} \sigma\left(X_{s}, \theta_{s}\right)}{V\left(X_{s}, \theta_{s}, Y_{s}\right)}\right|^{2} d s \\
& \leq \int_{0}^{t} \beta^{2} d s \leq \beta^{2} t .
\end{aligned}
$$

Next consider the quadratic variation of the local martingale term $M_{2}(t)$. Based on the following result presented in Kunita [15, page 323], and noting that the jump distribution $\pi$ is a probability measure that is, $\int_{\Gamma} \pi(d \gamma)=1$ we have

$$
\begin{aligned}
\left\langle M_{2}(t), M_{2}(t)\right\rangle & =\int_{0}^{t} \int_{\Gamma}\left(\log \left[\frac{V\left(X_{s-}+g\left(X_{s-}, \theta_{s-}, \gamma\right), \theta_{s-}, Y_{s-}\right)}{V\left(X_{s^{-}}, \theta_{s^{-}}, Y_{s-}\right)}\right]\right)^{2} \pi(d \gamma) d s \\
& \leq \max \left[\left(\log \rho_{1}\right)^{2},\left(\log \rho_{2}\right)^{2}\right] t
\end{aligned}
$$

On very similar lines, one can easily show that the quadratic variation of the local martingale term $M_{3}(t)$ is given by

$$
\left\langle M_{3}(t), M_{3}(t)\right\rangle \leq \max \left[\left(\log \bar{\rho}_{1}\right)^{2},\left(\log \bar{\rho}_{2}\right)^{2}\right] t .
$$


Thus by SLLN for local martingales (refer to Lipster and Shiryayev [16, page 140-141]), we can say that

$$
\limsup _{t \rightarrow \infty} \frac{M_{1}}{t}=\limsup _{t \rightarrow \infty} \frac{M_{2}}{t}=\limsup _{t \rightarrow \infty} \frac{M_{3}}{t}=0 .
$$

Thus from (3.2) and the above discussion, one can infer that

$$
\limsup _{t \rightarrow \infty} \frac{\log V(x, i, y)}{t} \leq-\alpha
$$

Thus, since by assumption $\alpha>0$, from the definition of almost sure exponential stability, the trivial solution to (1.1) is almost surely exponentially stable.

We now provide conditions under which the trivial solution to (1.1) is moment exponentially stable.

Theorem 3.2. Let $p, \alpha, \alpha_{1}, \alpha_{2}>0$. Assume that there exists a function $V(x, i, y) \in \mathcal{C}^{2,1}\left(\mathbb{R}^{r}, x, \mathbb{R}_{+}\right)$ such that

$$
\begin{gathered}
\alpha_{1}|x|^{p} \leq V(x, i, y) \leq \alpha_{2}|x|^{p}, \\
G V(x, i, y) \leq-\alpha|x|^{p} .
\end{gathered}
$$

Then,

$$
\limsup _{t \rightarrow \infty} \frac{1}{t} \log E\left|X_{t}\right|^{p} \leq \frac{-\alpha}{\alpha_{2}}\left|X_{0}\right|^{p}
$$

As a result the trivial solution of (1.1) is pth-moment exponentially stable under the conditions discussed above and the pth-moment Lyapunov exponent should not be greater than $-\alpha / \alpha_{2}$.

Proof. The proof is omitted as it is a simple extension of the Markov-modulated SDE case discussed in Mao [9].

In the next theorem, we provide criteria to connect these two seemingly disparate stabilty criteria. Specifically, we provide conditions under which the $p$ th-moment exponential stability for $p \geq 2$ always implies almost sure exponential stability for (1.1).

Theorem 3.3. Assume that there exists a positive constant $C$ such that for each $i \in \mathcal{X}$

$$
|b(x, i)| \vee|\sigma(x, i)| \vee|g(x, i, \gamma)| \leq C|x| \text {. }
$$

If for all $\mathrm{X}_{0}=x_{0} \in \mathbb{R}^{r}$,

$$
\limsup _{t \rightarrow \infty} \frac{1}{t} \log E\left(\left|X_{t}\right|^{p}\right) \leq-a
$$


then

$$
\limsup _{t \rightarrow \infty} \frac{1}{t} \log \left(\left|X_{t}\right|\right) \leq-\frac{a}{p} \text { a.s. }
$$

Then pth-moment exponential stability implies almost sure exponential stability.

We need the Burkholder-Davis-Gundy inequality which is detailed in the following remark below.

Remark 3.4. Let us recall that $[X]$ denotes the quadratic variation of a process say $X$, and $X_{t}^{*} \equiv \sup _{s \leq t}\left|X_{s}\right|$ is its maximum process. Then the Burkholder-Davis-Gundy theorem states that for any $1 \leq p<\infty$, there exist positive constants $c_{p}, C_{p}$ such that, for all local martingales $X$ with $X_{0}=0$ and stopping times $\tau$, the following inequality holds:

$$
c_{p} E\left[[X]_{\tau}^{p / 2}\right] \leq E\left[\left(X_{\tau}^{*}\right)^{p}\right] \leq C_{p} E\left[[X]_{\tau}^{p / 2}\right]
$$

Furthermore, for continuous local martingales, this statement holds for all $0<p<\infty$. For its proof refers to Theorem 3.28 page 166 in Karatzas and Shreve [17].

Proof of Theorem 3.3. Let $X_{0} \in \mathbb{R}^{r}$. Let $\epsilon$ be arbitrarily small positive number. By the definition of $p$ th-moment exponential stability of (3.15), there exists a constant $K$ such that

$$
E\left|X_{t}\right|^{p} \leq K \exp ^{-(a-\epsilon) t}, \quad t \geq 0
$$

Let $\delta>0$ be sufficiently small such that,

$$
5^{p} C^{p}\left(\delta^{p}+C_{p} \delta^{p / 2}\right)<\frac{1}{4} .
$$

From (1.1) we have

$$
\begin{aligned}
X_{t}= & X_{0}+\int_{0}^{t} b\left(X_{s}, \theta_{s}\right) d s+\int_{0}^{t} \sigma\left(X_{s}, \theta_{s}\right) d W_{s}+\int_{0}^{t} \int_{\Gamma} g\left(X_{s^{-}}, \theta_{s-}, \gamma\right) \widetilde{N}(d s, d \gamma) \\
& +\lambda \int_{0}^{t} \int_{\Gamma} g\left(X_{s^{-}}, \theta_{s-}, \gamma\right) \pi(d \gamma) d s .
\end{aligned}
$$

Noting that for $a, b, c, d, e \geq 0$

$$
\begin{aligned}
(a+b+c+d+e)^{p} \leq[5(a \vee b \vee c \vee d \vee e)]^{p} & =5^{p}\left(a^{p} \vee b^{p} \vee c^{p} \vee d^{p} \vee e^{p}\right) \\
& \leq 5^{p}\left(a^{p}+b^{p}+c^{p}+d^{p}+e^{p}\right)
\end{aligned}
$$


we have

$$
\begin{aligned}
E\left[\sup _{(k-1) \delta \leq t \leq k \delta}\left|X_{t}\right|^{p}\right] \leq & 5^{p} E\left[\left|X_{(k-1) \delta}\right|^{p}\right]+5^{p} E\left(\int_{(k-1) \delta}^{k \delta}\left|b\left(X_{s}, \theta_{s}\right)\right| d s\right)^{p} \\
& +5^{p} E\left(\sup _{(k-1) \delta \leq t \leq k \delta} \int_{(k-1) \delta}^{t}\left|\sigma\left(X_{s}, \theta_{s}\right) d W_{s}\right|^{p}\right) \\
& +5^{p} E\left(\sup _{(k-1) \delta \leq t \leq k \delta} \int_{(k-1) \delta}^{t} \int_{\Gamma}\left|g\left(X_{s-}, \theta_{s^{-}}, \gamma\right) \widetilde{N}(d s, d \gamma)\right|^{p}\right) \\
& +5^{p} \lambda^{p} E\left(\int_{(k-1) \delta}^{k \delta} \int_{\Gamma}\left|g\left(X_{s^{-}}, \theta_{s^{-}}, \gamma\right)\right| \pi(d \gamma) d s\right)^{p} .
\end{aligned}
$$

Noting that $\int_{\Gamma} \pi(d \gamma)=1$, we have

$$
\begin{gathered}
E\left(\sup _{(k-1) \delta \leq t \leq k \delta} \int_{(k-1) \delta}^{t} \int_{\Gamma}\left|g\left(X_{s^{-}}, \theta_{s^{-}}, \gamma\right) \widetilde{N}(d s, d \gamma)\right|^{p}\right) \\
\leq C_{p} E\left(\int_{(k-1) \delta}^{k \delta}\left|g\left(X_{s^{-}}, \theta_{s^{-}}, \gamma\right)\right|^{2} d s\right)^{(p / 2)} \\
\leq C_{p} E\left(\delta \sup _{(k-1) \delta \leq s \leq k \delta}\left|g\left(X_{s^{-}}, \theta_{s^{-}}, \gamma\right)\right|^{2}\right)^{(p / 2)} \\
\leq C_{p} C^{p} \delta^{p / 2} E\left[\sup _{(k-1) \delta \leq s \leq k \delta}\left|X_{s}\right|^{p}\right] .
\end{gathered}
$$

Similarly,

$$
\begin{aligned}
E\left(\left|\int_{(k-1) \delta}^{k \delta} \int_{\Gamma}\right| g\left(X_{s^{-}}, \theta_{s^{-}}, \gamma\right) \pi(d \gamma) d s\right)^{p} & \leq E\left[\delta \sup _{(k-1) \delta \leq s \leq k \delta}\left|g\left(X_{s^{-}}, \theta_{s^{-}}, \gamma\right)\right|\right]^{p} \\
& \leq C^{p} \delta^{p} E\left(\sup _{(k-1) \delta \leq s \leq k \delta}\left|X_{s}\right|^{p}\right) .
\end{aligned}
$$

From (3.15), one can easily show that

$$
\begin{gathered}
E\left[\left|X_{(k-1) \delta}\right|^{p}\right] \leq K \exp ^{-(a-\epsilon)(k-1) \delta}, \\
E\left(\int_{(k-1) \delta}^{k \delta}\left|b\left(X_{s}, \theta_{s}\right)\right| d s\right)^{p} \leq C^{p} \delta^{p} E\left[\sup _{(k-1) \delta \leq s \leq k \delta}\left|X_{s}\right|^{p}\right], \\
E\left(\sup _{(k-1) \delta \leq t \leq k \delta} \int_{(k-1) \delta}^{t}\left|\sigma\left(X_{s}, \theta_{s}\right)\right| d W_{s}\right)^{p} \leq C_{p} C^{p} \delta^{p / 2} E\left[\sup _{(k-1) \delta \leq s \leq k \delta}\left|X_{s}^{p}\right|\right] .
\end{gathered}
$$


Hence, substituting (3.20)-(3.23) in (3.19), we obtain

$$
\begin{aligned}
& E\left[\sup _{(k-1) \delta \leq t \leq k \delta}\left|X_{t}\right|^{p}\right]\left(1-5^{p}\left(C^{p} \delta^{p}+C_{p} C^{p} \delta^{p / 2}+C_{p} C^{p} \delta^{p / 2}+C^{p} \delta^{p}\right)\right) \\
& \leq K 5^{p} \exp ^{-(a-e)(k-1) \delta} .
\end{aligned}
$$

From (3.16) we obtain that

$$
E\left[\sup _{(k-1) \delta \leq t \leq k \delta}\left|X_{t}\right|^{p}\right] \leq 2 \times 5^{p} K \exp ^{-(a-\epsilon)(k-1) \delta},
$$

and utilizing the Borel-Cantelli Lemma as in Mao [9] we deduce the desired implication that pth-moment stability implies almost sure exponential stability.

\section{Examples}

We now provide some simple examples to illustrate both the almost surely exponential stability and moment exponential stability. We start with an example on almost surely exponential stability.

Consider a two state semi-Markov modulated Jump-diffusion problem with $X_{t} \in \mathbb{R}^{r}$ and $V\left(X_{t}, i, Y_{t}\right)=\left|X_{t}\right|$ where the generator matrix is given by

$$
Q=\left|\begin{array}{cc}
-2 & 2 \\
1 & -1
\end{array}\right|
$$

Let the holding time in each regime be assumed to follow $f(y \mid i)=\lambda_{i} e^{-\lambda_{i} y}, y>0, i \in\{1,2\}$. Note that with the choice of the holding time distribution, the sMMJD collapses to the MMJD case in which case the generator $G$ acting on $V(x, i, y)$ is given by

$$
\begin{aligned}
G V(x, i, y)= & \frac{1}{2} \operatorname{trace}\left[\left(\frac{I}{|x|}-\frac{x x^{\prime}}{|x|^{3}}\right) \sigma(x, i) \sigma^{\prime}(x, i)\right] \\
& +\frac{x^{\prime}}{|x|} b(x, i)+\lambda \int_{\Gamma}[|x+g(x, i, \gamma)|-|x|] \pi(d \gamma) .
\end{aligned}
$$

Now from Assumption 2.1 as

$$
\begin{aligned}
|\sigma(x, i)| & =|\sigma(x, i)-\sigma(0, i)| \leq C|x| \\
|b(x, i)| & =|b(x, i)-b(0, i)| \leq C|x|
\end{aligned}
$$


and $|g(x, i, \gamma)| \leq C|x|$, we have

$$
\begin{aligned}
G V(x, i, y) & \leq C|x|+C|x|+\lambda(2+C)|x| \\
& =(2 C+\lambda C+2 \lambda)|x| .
\end{aligned}
$$

If we choose $C$ and $\lambda$ such that for any $x \in \mathbb{R}^{r}-\{0\}$, there exists $\left.\alpha:=(2+\lambda) C+2 \lambda\right) \geq 0$ such that $G \log V(x, i, y) \leq-\alpha$. Also $\left|\nabla_{x} V(x, i, y)^{\prime} \sigma(x, i) / V(x, i, y)\right| \leq C$. Similarly, if there exist a positive constant $\beta$ such that for any $x \in \mathbb{R}^{r}, C \leq \beta$, then $\left|\nabla_{x} V(x, i, y)^{\prime} \sigma(x, i) / V(x, i, y)\right| \leq \beta$. If there exist constants $\rho_{1}$ and $\rho_{2}$ such that $\rho_{1} \leq g(x, i, \gamma) \leq \rho_{2}$ for any $x \in \mathbb{R}^{r}, i \in \mathcal{X}$ and $\gamma \in \Gamma$, then it is easy to see that $\left(\rho_{1}\right) \leq(V(x+g(x, i, \gamma), i, y) / V(x, i, y)) \leq\left(\rho_{2}\right)$. Thus in brief for certain conditions on the growth of the drift, diffusion and the integrand of the jump component of the process given by (1.1), we satisfy the conditions of Theorem 3.1 for the solution to (1.1) to be almost surely exponentially stable.

We next provide a simple example to illustrate Theorem 3.2. Consider that $x \in \mathbb{R}$ and $V(x, i, y)=x^{2}$. Also assume that the conditional holding time distribution be $f(y \mid i)=\lambda_{i} e^{-\lambda_{i} y}$ for $i \in\{1,2\}$. Let $g(x, i, \gamma)=x, \lambda_{i}=1, b(x, i)=a_{1} x, \sigma(x, i)=a_{2} x$ for $i \in\{1,2\}$. Then from (2.4) we have $G V(x, i, y)=\left(2 a_{1}+a_{2}+3\right) x^{2}$. If $2 a_{1}+a_{2}+3<0$ and $x \neq 0$, then condition (ii) of Theorem 3.2 for $p=2$ is satisfied. Moreso if we assume that there exist constants $\alpha_{1}$ and $\alpha_{2}$ such that $\alpha_{1}|x|^{2} \leq V(x, i, y) \leq \alpha_{2}|x|^{2}$ is true, then condition (i) of Theorem 3.2 is satisfied. Thus both conditions (i) and (ii) now guarantee that the solution of (1.1) is moment exponentially stable.

Next we discuss the issue of stochastic stabilization and destabilization of nonlinear systems.

\section{Stochastic Stabilization and Destabilization of Nonlinear Systems}

We now investigate the stability of the nonlinear deterministic system of differential equations given by the following dynamics:

$$
\frac{d X_{t}}{d t}=f\left(X_{t}\right)
$$

on $t \geq 0$ with $X_{0}=x_{0} \in \mathbb{R}^{r}$ where $f(x): \mathbb{R}^{r} \rightarrow \mathbb{R}^{r}$ is locally Lipschitz continuous and furthermore there exists some constant $K>0$ such that $|f(x)| \leq K|x|$ for all $x \in \mathbb{R}^{r}$. When perturbed by noise, the nonlinear system (5.1) is either stable if it originally unstable, in the sense that by adding noise we can force the solution of the stochastic differential equation to converge to the trivial solution as time increases indefinitely. This is the aim of stochastic stabilization. Likewise if our original system in stable, then this system is said to destabilize when perturbed by noise if the sample paths of the process escapes to infinity almost surely instead of converging to the trivial solution as time tends to infinity. This is termed as stochastic destabilization. Consequently, the system then becomes what is known as unstable. Mao [9] and Applebaum and Siakalli [18] have established a general theory of stochastic stabilization/destabilization of (5.1) using a Brownian motion and the general Levy process, respectively. However, no specific work has been done so far for the case where $X_{t}$ is an SMMJD. In this paper we focus on the first-order nonlinear system of ODEs that is perturbed by an SMMJD. In the following section, we show that an unstable linear system counterpart 
of (5.1) wherein $d X_{t} / d t=a X_{t}$ for $a>0$ can be stabilized just by the addition of a jump component to the dynamics of the one-dimensional process $X_{t}$. We observe that such a jumpstabilized system of DEs cannot be destabilized by further addition of a Brownian motion. On the contrary, we show that such a jump-stabilized nonlinear system of differential equations can surprisingly be destabilized by addition of Brownian motion if the dimension of the state space is at least two. Before we go into the proofs of these statements, we begin by mentioning the key dynamics of the sMMJD process $\left\{X_{t}, t \geq 0\right\}$ and some assumptions that follow. Suppose we have an $m$-dimensional standard $\mathcal{F}_{T}$-adapted Brownian motion process $B=\left(B_{1}(t), \ldots, B_{m}(t)\right)$ for each $t \geq 0$. The system (5.1) is perturbed by the following sMMJD dynamics of $X_{t}$ given by

$$
d X_{t}=f\left(X_{t}\right) d t+\sum_{k=1}^{m} G_{k}\left(\theta_{t}\right) X_{t} d B_{k}(t)+\lambda \int_{\Gamma} D\left(\theta_{t-}, \gamma\right) X_{t-} N(d t, d \gamma) \quad \forall t \geq 0
$$

where $G_{k}(i)$ is $\mathbb{R}^{r \times r}$ for each $i \in \mathcal{X}$. Likewise $D(i, \gamma)$ is an $\mathbb{R}^{r \times r}$-valued matrix for each $i \in \mathcal{X}$ and $\gamma \in \Gamma \subset \mathbb{R}^{r}-\{0\}$. We refer to a system (5.1) perturbed by the dynamics of $X_{t}$ as in (5.2) as just a perturbed system. We make the following key assumption that remains valid until the end of this section.

Assumption 5.1. Assume that for each $i \in \mathcal{X}$ and $\gamma \in \Gamma$ we have $\int_{\Gamma}\left(\|D(i, \gamma)\| \vee\|D(i, \gamma)\|^{2}\right)$ $\pi(d \gamma)<\infty$ and that $D(i, \gamma)$ does not have an eigenvalue equal to $-1 \pi$ almost surely.

In the following, we will establish the conditions on the coefficients of (5.2) for the trivial solution of the perturbed system to be almost surely exponentially stable. In particular, this surprisingly demonstrates that the jump process can have a stabilizing effect, as for the Brownian motion part as has been shown by Mao [9]. We state this formally as one of our main theorems.

Theorem 5.2. Assume that Assumption 5.1 holds. Suppose that the following conditions are satisfied for $a(i)>0, b(i) \geq 0$ :

(i) $\sum_{k=1}^{m}\left|G_{k}(i) x\right|^{2} \leq a(i)|x|^{2}$,

(ii) $\sum_{k=1}^{m}\left|x^{\prime} G_{k}(i) x\right|^{2} \geq b(i)|x|^{4}$ for each $i \in \mathcal{X}$ and $x \in \mathbb{R}^{r}$.

Then the sample Lyapunov exponent of the solution of (5.2) exists and satisfies $\lim \sup _{t \rightarrow \infty} \log \left|X_{t}\right| \leq$ $K-\sum_{i \in X}[(b(i)-a(i) / 2-\lambda \log (1+\|D(i, \gamma)\|))] v_{i}$ for any $X_{0} \neq 0$. If $-K+\sum_{i \in \mathcal{X}}[b(i)-a(i) / 2-\lambda \log (1+$ $\|D(i, \gamma)\|)] v_{i}>0$, then the trivial solution to the system in (5.2) is almost surely exponentially stable.

Proof.

Step 1. Define $V(x, i, y)=\log |x|$ for all $i \in \mathcal{X}$. As $V(x, i, y)$ is independent of states $i$ and $y$, the following terms in (2.4) are zero:

$$
\begin{gathered}
\frac{f(y \mid i)}{1-F(y \mid i)} \sum_{j \neq i, j \in \mathcal{X}} p_{i j}[V(x, j, 0)-V(x, i, y)]=0, \\
\frac{\partial V(x, i, y)}{\partial y}=0 .
\end{gathered}
$$


Hence as an application of the generalized Ito's formula, we have for $t>0$

$$
\begin{aligned}
\log \left|X_{t}\right|= & \log \left|X_{0}\right|+\int_{0}^{t} \frac{X_{s}^{\prime}}{\left|X_{s}\right|^{2}} f\left(X_{s}\right) d s+\frac{1}{2} \sum_{k=1}^{m} \int_{0}^{t}\left[\frac{\left|G_{k}\left(\theta_{s}\right) X_{s}\right|^{2}}{\left|X_{s}\right|^{2}}-\frac{2\left|X_{s}^{\prime} G_{k}\left(\theta_{s}\right) X_{s}\right|^{2}}{\left|X_{s}\right|^{4}}\right] d s \\
& +\lambda \int_{0}^{t} \int_{\Gamma} \log \left(\frac{\left|X_{s-}+D\left(\theta_{s-}, \gamma\right) X_{s-}\right|}{\left|X_{s-}\right|}\right) \pi(d \gamma) d s+M_{1}(t)+M_{2}(t),
\end{aligned}
$$

where $M_{1}(t)=\left.\sum_{k=1}^{m} \int_{0}^{t}\left|X_{s}^{\prime} G_{k}\left(\theta_{s}\right) X_{s} /\right| X_{s}\right|^{2} \mid d B_{k}(s)$ and $M_{2}(t)=\int_{0}^{t} \int_{\Gamma} \log \left(\left(\left|X_{s^{-}}+D\left(\theta_{s^{-}}, \gamma\right) X_{s^{-}}\right|\right) /\right.$ $\left.\left|X_{s-}\right|\right) \widetilde{N}(d s, d \gamma)$ are the two local martingale terms.

Step 2. Consider now the quadratic variation of the two martingale terms. From Ito's isometry and noting that

$$
\begin{aligned}
\frac{\left|X_{s}^{\prime} G_{k}\left(\theta_{s}\right) X_{s}\right|^{2}}{\left|X_{s}\right|^{4}} & =\frac{\left|X_{s}^{\prime}\left(G_{k}^{\prime}\left(\theta_{s}\right)+G_{k}\left(\theta_{s}\right)\right) X_{s}\right|^{2}}{4\left|X_{s}\right|^{4}} \\
& \leq \rho\left(G_{k}\left(\theta_{s}\right)\right)^{2},
\end{aligned}
$$

where $\rho\left(G_{k}\left(\theta_{s}\right)\right)$ is the spectral radius of the symmetric $r \times r$ matrix $\left(G_{k}\left(\theta_{s}\right)+G_{k}^{\prime}\left(\theta_{s}\right)\right) / 2$,

$$
\left\langle M_{1}(t), M_{1}(t)\right\rangle \leq \sum_{k=1}^{m} \int_{0}^{t} \frac{\left|X_{s}^{\prime} G_{k}\left(\theta_{s}\right) X_{s}\right|^{2}}{\left|X_{s}\right|^{4}} d s \leq t m \max _{1 \leq k \leq m, i=1, \ldots, M} \rho\left(G_{k}(i)\right) .
$$

Next, the quadratic variation of the process $M_{2}(t)$ is given by

$$
\begin{aligned}
\left\langle M_{2}(t), M_{2}(t)\right\rangle & =2 \int_{\Gamma} \int_{0}^{t} \log \left[\frac{\left(\left|X_{s-}+D\left(\theta_{s-}, \gamma\right) X_{s-}\right|\right)}{\left|X_{s-}\right|}\right] d s \pi(d \gamma) \\
& \leq 2 t \log \left(1+\max _{1 \leq i \leq M}\|D(i, \gamma)\|\right) .
\end{aligned}
$$

Step 3. We work with the rest of the terms in the following way:

$$
\limsup _{t \rightarrow \infty}\left|\frac{1}{t} \int_{0}^{t} \frac{X_{s}^{\prime} f\left(X_{s}\right)}{\left|X_{s}\right|^{2}} d s\right| \leq K,
$$


also

$$
\begin{aligned}
& \limsup _{t \rightarrow \infty} \frac{1}{t} \int_{0}^{t} \frac{1}{2}\left[\sum_{k=1}^{m}\left(\frac{\left|G_{k}\left(\theta_{s}\right) X_{s}\right|^{2}}{\left|X_{s}\right|^{2}}-\frac{2\left|X_{s} G_{k}\left(\theta_{s}\right) X_{s}\right|^{2}}{\left|X_{s}\right|^{4}}\right)\right] d s \\
& \leq \frac{1}{t} \sum_{i \in \mathcal{X}} \int_{0}^{t}\left[\frac{a(i)}{2}-b(i)\right] \mathbb{I}_{\theta_{s}=i} d s \\
& \leq \sum_{i \in \mathcal{X}}\left[\frac{a(i)}{2}-b(i)\right] v_{i,} \\
& \limsup _{t \rightarrow \infty} \frac{\lambda}{t} \int_{0}^{t} \int_{\Gamma} \log \left(\frac{\left|X_{s-}+D\left(\theta_{s-}, \gamma\right) X_{s-}\right|}{\left|X_{s-}\right|}\right) \pi(d \gamma) d s \\
& \leq \lambda \sum_{i \in \mathcal{X}} \log (1+\|D(i, \gamma)\|) v_{i} .
\end{aligned}
$$

Thus, $\lim \sup _{t \rightarrow \infty}(1 / t) \log \left|X_{t}\right|<0$ if $K+\sum_{i \in \mathcal{X}}[(a(i) / 2-b(i)+\lambda \log (1+\|D(i, \gamma)\|))] v_{i}<0$.

Remark 5.3. Consider a 1-D sMMJD with the dynamics

$$
d X_{t}=a X_{t} d t+b(i) X_{t} d B_{t}+c(i, \gamma) X_{t} d \widetilde{N}_{t}
$$

where $b(x, i)>0$ and $c(i, \gamma)>-1$ for each $x \in \mathbb{R}, i \in\{1, \ldots, M\}$ and $\gamma \in \Gamma$. $B_{t}$ is a 1D Brownian motion and $\left\{\widetilde{N}_{t}, t \geq 0\right\}$ is a compensated Poisson process with $\widetilde{N}_{t}=N_{t}-\lambda t$, where $\lambda>0$ is the intensity of the Poisson process. Assume that the processes $B_{t}$ and $N_{t}$ are independent. Then one can show from the SLLN for a Brownian motion and for a Poisson process (refer to Applebaum [13]) that for each $i \in\{1,2, \ldots, M\}$

$$
\limsup _{t \rightarrow \infty} \frac{1}{t} \log \left|X_{t}\right|=a+\left[-\lambda c(i, \gamma)-\frac{1}{2} b^{2}(i)+\lambda \log (1+c(i, \gamma))\right]<0 \quad \text { a.s. }
$$

Note that $b^{2}(i) \geq 0$ for all $i \in \mathcal{X}$ and has a negative sign attached to it. Hence when the onedimensional perturbed system $d X_{t} / d t=a X_{t}$ for $a>0$ is stabilized by the addition of a jump process infact can never be destabilized by the addition of a Brownian motion. An interesting question we may ask here is: can the same inference hold true in higher dimensions? The answer is surprisingly no. In the following theorem, we show that for a state space of dimension greater than or equal to two, an unstable nonlinear system of differential equation stabilized by a jump component can still be destabilized by the addition of the Brownian motion. This surprising phenomenon was also observed by Applebaum and Siakalli [19] for the Levy process case.

To prove this assertion, let us now consider system of nonlinear differential equation (5.1) stabilized by (5.2) but with $G_{k}(i)=0$ for each $i=1, \ldots, M$ and $k=1, \ldots, m$. We now show that it gets destabilized by further addition of the $m$-dimensional Brownian motion to (5.1). This corresponds to $G_{k}(i) \neq 0$ for each $i=1, \ldots, M$ and $k=1, \ldots, m$. 
Theorem 5.4. Assume that matrix $D$ is an $r \times r$ symmetric positive definite matrix. Now let

$$
\begin{aligned}
& \text { (i) } \sum_{k=1}^{m}\left|G_{k}(i) x\right|^{2} \geq a(i)|x|^{2}, \\
& \text { (ii) } \sum_{k=1}^{m}\left|x^{\prime} G_{k}(i) x\right|^{2} \leq b(i)|x|^{4},
\end{aligned}
$$

for $a(i)>0, b(i) \geq 0$ for each $i \in \mathcal{X}, x \in \mathbb{R}^{r}$. Hence $\liminf _{t \rightarrow \infty}(1 / t) \log \left|X_{t}\right| \geq-K+\sum_{i \in \mathcal{X}}[(a(i) / 2-$ $\left.\left.b(i)+\lambda \log \left(1+\min _{1 \leq i \leq M}\|D(i, \gamma)\|\right)\right)\right] v_{i}$ for any $X_{0} \neq 0$. In particular if $-K+\sum_{i \in X}[a(i) / 2-b(i)+$ $\left.\lambda \log \left(1+\min _{1 \leq i \leq M}\|D(i, \gamma)\|\right)\right] v_{i}>0$, then the trivial solution of $(5.2)$, tends to be infinity almost surely exponentially fast.

Proof. Fix $X_{0} \neq 0$. From Lemma 2.2, $X_{t} \neq 0$ for all $t \geq 0$. Applying Ito's lemma to $\log \left|X_{t}\right|$, for $t>0$ and for each $i \in \mathcal{X}$,

$$
\begin{aligned}
\log \left|X_{t}\right|= & \log \left|X_{0}\right|+\int_{0}^{t} \frac{X_{s}^{\prime}}{\left|X_{s}\right|^{2}} f\left(X_{s}\right) d s+\frac{1}{2} \sum_{k=1}^{m} \int_{0}^{t}\left[\frac{\left|G_{k}\left(\theta_{s}\right) X_{s-}\right|^{2}}{\left|X_{s-}\right|^{2}}-\frac{2\left|X_{s-} G_{k}\left(\theta_{s}\right) X_{s-}\right|^{2}}{\left|X_{s-}\right|^{4}}\right] d s \\
& +\lambda \int_{0}^{t} \int_{\Gamma} \log \left(\frac{\left(\left|X_{s-}+D\left(\theta_{s-}=i, Y\right) X_{s-}\right|\right)}{\left|X_{s-}\right|}\right) \pi(d \gamma) d s+M_{1}(t)+M_{2}(t),
\end{aligned}
$$

where $M_{1}(t)=\sum_{k=1}^{m} \int_{0}^{t}\left(\left|X_{s-}^{\prime} G_{k}\left(\theta_{s}\right) X_{s-}\right| /\left|X_{s-}\right|^{2}\right) d B_{k}(s)$ and $M_{2}(t)=\int_{\Gamma} \int_{0}^{t} \log \left(\left(\mid X_{s^{-}}+D\left(\theta_{s_{-}}=\right.\right.\right.$ $\left.\left.i, Y) X_{s-} \mid\right) /\left|X_{s-}\right|\right) \widetilde{N}(d s, d \gamma)$ are the two local martingale terms. Now using methodology similar to Theorem 5.2 we find

$$
\liminf _{t \rightarrow \infty} \frac{1}{t} \log \left|X_{t}\right| \geq-K+\sum_{i \in \mathcal{X}}\left[\left(\frac{a(i)}{2}-b(i)+\lambda \log \left(1+\min _{1 \leq i \leq M}\|D(i, \gamma)\|\right)\right)\right] v_{i}
$$

for any $X_{0} \neq 0$. In particular, if $-K+\sum_{i \in X}\left[a(i) / 2-b(i)+\lambda \log \left(1+\min _{1 \leq i \leq M}\|D(i, \gamma)\|\right)\right] v_{i}>0$, then the trivial solution of the $X_{t}$-perturbed system given by (5.2) tends to be infinity almost surely exponentially fast.

\section{Concluding Remarks}

We presented conditions under which the solution of a semi-Markov Modulated jump diffusion is almost surely exponentially stable and moment exponentially stable. We also provide conditions that connect these two notions of stability. We further determine the conditions under which the trivial solution of the sMMJD-perturbed nonlinear system of differential equation $d X_{t} / d t=f\left(X_{t}\right)$ is almost surely exponentially stable. We show that an unstable deterministic system can be stabilized by adding jumps. Such jump stabilized system, however, can get de-stabilized by Brownian motion if the dimension of the state space is at least two.

\section{Acknowledgments}

The author is very grateful to the anonymous referees and the editor for their careful reading, valuable comments, and helpful suggestions, which have helped him to improve the presentation of this paper significantly. 


\section{References}

[1] L. Arnold, Differential Equations: Theory and Applications, John Wiley \& Sons, New York, NY, USA, 1972.

[2] R. Z. Khasminskii, Stochastic Stability of Differential Equations, vol. 7, Sijthoff and Noordhoff, Alphen aan den Rijn, The Netherlands, 1980.

[3] G. S. Ladde and V. Lakshmikantham, Random Differential Inequalities, Academic Press, New York, NY, USA, 1980.

[4] A. Deshpande and M. K. Ghosh, "Risk minimizing option pricing in a regime switching market," Stochastic Analysis and Applications, vol. 26, no. 2, pp. 313-324, 2008.

[5] F. B. Hanson, Applied Stochastic Processes and Control for Jump-Diffusions, vol. 13 of Advances in Design and Control, Society for Industrial and Applied Mathematics (SIAM), Philadelphia, Pa, USA, 2007.

[6] Y. Ji and H. J. Chizeck, "Controllability, stabilizability, and continuous-time Markovian jump linear quadratic control," Institute of Electrical and Electronics Engineers, vol. 35, no. 7, pp. 777-788, 1990.

[7] M. Mariton, Jump Linear Systems in Automatic Control, Marcel Dekker, New York, NT, USA, 1990.

[8] G. K. Basak, A. Bisi, and M. K. Ghosh, "Stability of a random diffusion with linear drift," Journal of Mathematical Analysis and Applications, vol. 202, no. 2, pp. 604-622, 1996.

[9] X. Mao, "Stability of stochastic differential equations with Markovian switching," Stochastic Processes and their Applications, vol. 79, no. 1, pp. 45-67, 1999.

[10] G. Yin and F. Xi, "Stability of regime-switching jump diffusions," SIAM Journal on Control and Optimization, vol. 48, no. 7, pp. 4525-4549, 2010.

[11] M. K. Ghosh and A. Goswami, "Risk minimizing option pricing in a semi-Markov modulated market," SIAM Journal on Control and Optimization, vol. 48, no. 3, pp. 1519-1541, 2009.

[12] F. Xi, "Asymptotic properties of jump-diffusion processes with state-dependent switching," Stochastic Processes and their Applications, vol. 119, no. 7, pp. 2198-2221, 2009.

[13] D. Applebaum, Léry Processes and Stochastic Calculus, Cambridge University Press, Cambridge, UK, 2nd edition, 2009.

[14] N. Ikeda and S. Watanabe, Stochastic Differential Equations and Diffusion Processes, vol. 24 of NorthHolland Mathematical Library, North-Holland, Amsterdam, The Netherlands, 2nd edition, 1989.

[15] H. Kunita, "Stochastic differential equations based on Lévy processes and stochastic flows of diffeomorphisms," in Real and Stochastic Analysis, M. M. Rao, Ed., pp. 305-373, 2004.

[16] R. S. Lipster and A. N. Shiryayev, Theory of Martingales, Horwood, Chichester, UK, 1989.

[17] I. Karatzas and S. E. Shreve, Brownian Motion and Stochastic Calculus, Springer, New York, NY, USA, 2nd edition, 1991.

[18] D. Applebaum and M. Siakalli, "Asymptotic stability of stochastic differential equations driven by Lévy noise," Journal of Applied Probability, vol. 46, no. 4, pp. 1116-1129, 2009.

[19] D. Applebaum and M. Siakalli, "Stochastic stabilization of dynamical systems using Lévy noise," Stochastics and Dynamics, vol. 10, no. 4, pp. 509-527, 2010. 


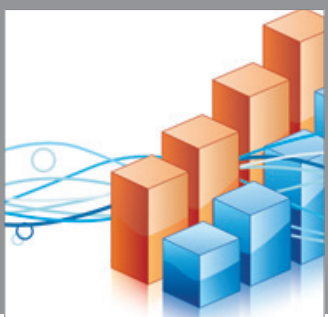

Advances in

Operations Research

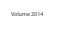

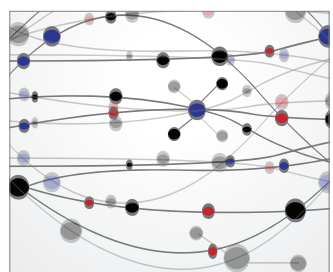

\section{The Scientific} World Journal
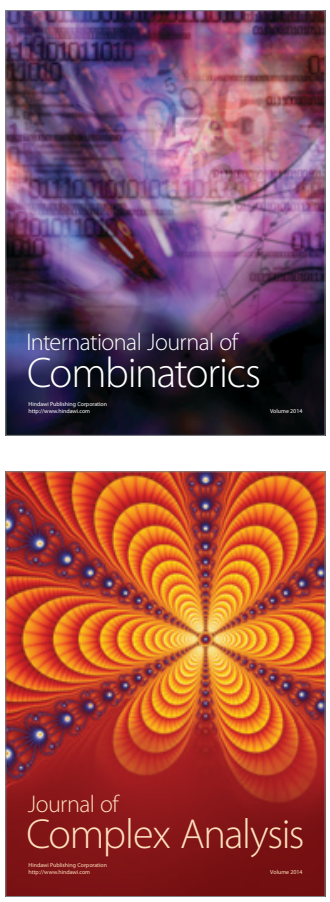

International Journal of

Mathematics and

Mathematical

Sciences
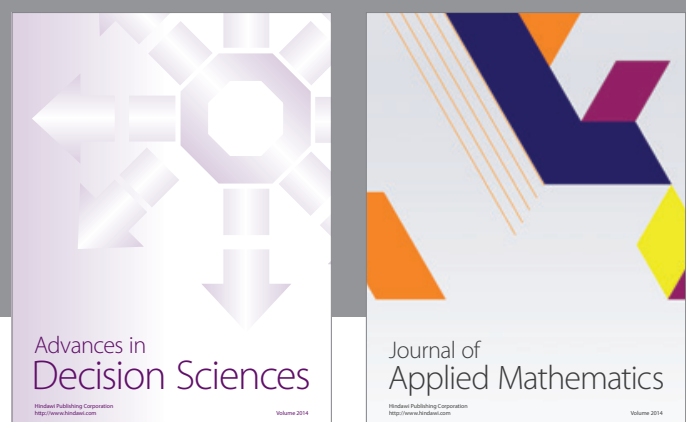

Journal of

Applied Mathematics
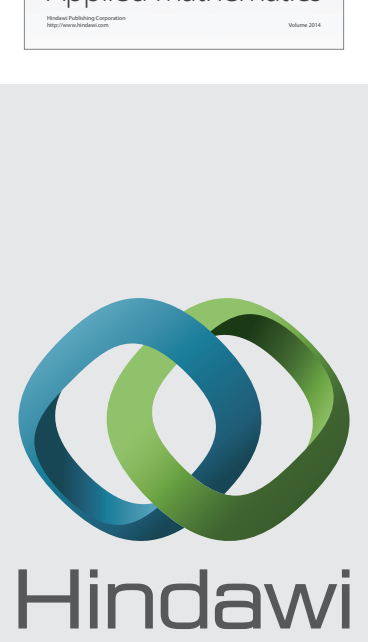

Submit your manuscripts at http://www.hindawi.com
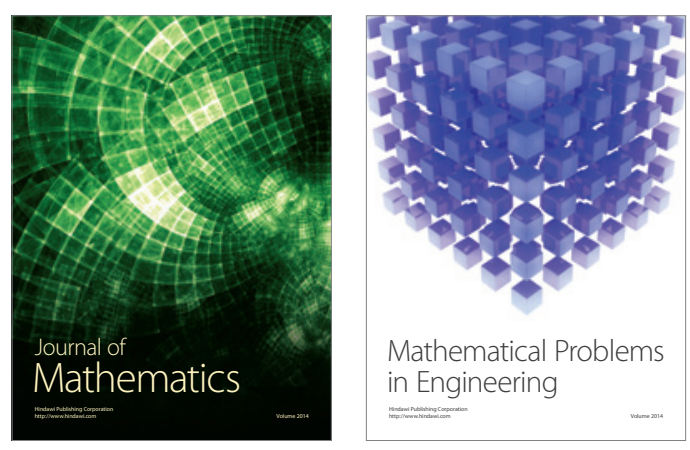

Mathematical Problems in Engineering
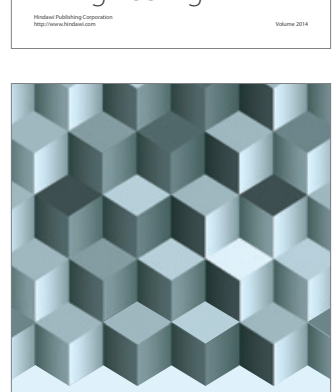

Journal of

Function Spaces
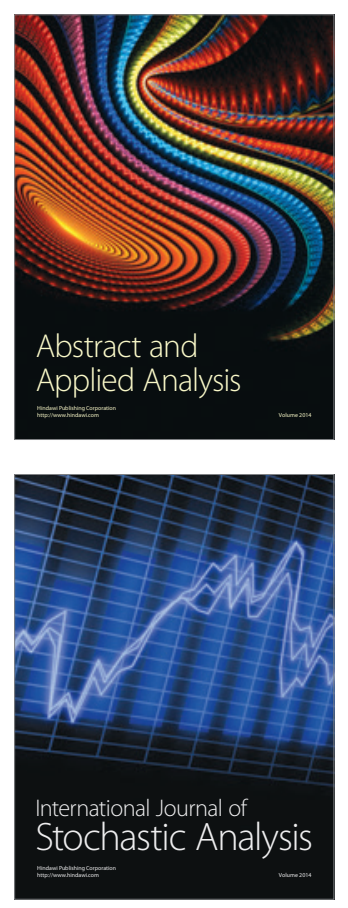

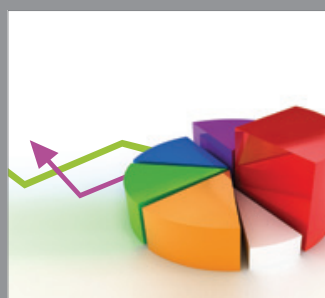

ournal of

Probability and Statistics

Promensencen
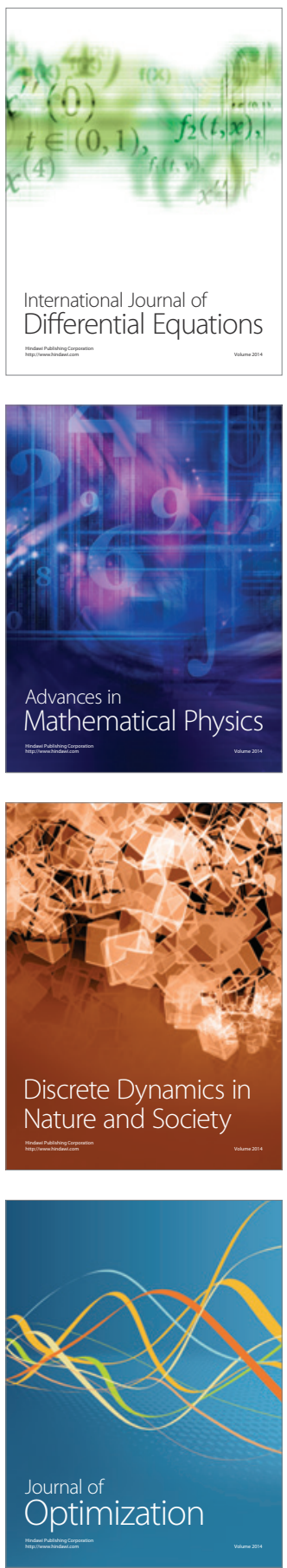